Hoa Q. Do, Marc Wittlich, Julian M. Glück, Luis Möckel, Dieter Willbold, Bernd W. Koenig and Henrike Heise*

\title{
Full-length Vpu and human CD4(372-433) in phospholipid bilayers as seen by magic angle spinning NMR
}

\begin{abstract}
HIV-1 Vpu and CD4(372-433), a peptide comprising the transmembrane and cytoplasmic domain of human CD4, were recombinantly expressed in Escherichia coli, uniformly labeled with ${ }^{13} \mathrm{C}$ and ${ }^{15} \mathrm{~N}$ isotopes, and separately reconstituted into phospholipid bilayers. Highly resolved dipolar cross-polarization (CP)-based solid-state NMR spectra of the two transmembrane proteins were recorded under magic angle sample spinning. Partial assignment of ${ }^{13} \mathrm{C}$ resonances was achieved. Site-specific assignments were obtained for 13 amino acid residues of CD4(372-433) and two Vpu residues. Additional amino acid type-specific assignments were achieved for 10 amino acid spin systems for both CD4(372-433) and Vpu. Further, structural flexibility was probed with different dipolar recoupling techniques, and the correct insertion of the transmembrane domains into the lipid bilayers was confirmed by proton spin diffusion experiments.
\end{abstract}

Keywords: CD4; HIV-1 Vpu; lipid-edited solid-state NMR spectroscopy; POPC lipid bilayers.

*Corresponding author: Henrike Heise, Institute of Complex Systems (ICS-6), Research Center Jülich, D-52425 Jülich, Germany; and Institute of Physical Biology, Heinrich-Heine-University Düsseldorf, Universitätsstrasse 1, D-40225 Düsseldorf, Germany, e-mail: h.heise@fz-juelich.de

Hoa Q. Do, Marc Wittlich, Julian M. Glück, Luis Möckel, Dieter Willbold and Bernd W. Koenig: Institute of Complex Systems (ICS-6), Research Center Jülich, D-52425 Jülich, Germany; and Institute of Physical Biology, Heinrich-Heine-University Düsseldorf, Universitätsstrasse 1, D-40225 Düsseldorf, Germany

\section{Introduction}

The T-cell coreceptor CD4 is a monotopic integral membrane protein. Human CD4 comprises 433 amino acid residues. It consists of a large glycosylated N-terminal extracellular domain, a transmembrane (TM) helix spanning residues 373-395 and a small cytoplasmic domain containing residues 396-433 (Maddon et al., 1985, 1987). In HIV-1 infection, CD4 serves as the main receptor for the virus on the surface of CD4-positive immune cells.

Vpu (Cohen et al., 1988; Strebel et al., 1988, 1989; Chengmayer et al., 1990; Klimkait et al., 1990) is a small, integral membrane protein encoded in the HIV-1 genome. It consists of 81 residues, comprising one TM helix (residues 8-25) (Park et al., 2003, 2006a) and a cytoplasmic region (residues 26-81) that can be subdivided in a short stretch rich in basic amino acids and a very acidic cytoplasmic tail (residues 39-81). Two additional helices are formed in the cytoplasmic region of $\mathrm{Vpu}$ in the presence of a membrane or a suitable membrane mimic (Ma et al., 2002; Wittlich et al., 2009). However, the exact length and position of these helices depend on the specific properties of the membrane and the HIV-1 strain-specific Vpu sequence. As an accessory protein, Vpu is not directly involved in the replication of the viral genome. Two major functions of Vpu in the viral life cycle have been identified (Schubert et al., 1996a). First, it enhances the release of progeny virions from infected cells. Second, it is involved in downregulation of CD4 newly synthesized in the endoplasmic reticulum (ER) of HIV-1-infected cells (Dube et al., 2010).

Enhancement of virus release by Vpu has been discussed as a consequence of the ability of the TM domain of $\mathrm{Vpu}$ to form oligomeric, cation-selective ion channels in lipid bilayers (Maldarelli et al., 1993; Schubert et al., 1996b; Cordes et al., 2002). Vpu was classified as a class IA viroporin (Fischer et al., 2012; Luis Nieva et al., 2012). Recently it was found that Vpu antagonizes tetherin, a cellular antiviral defense factor that cross-links budding virions to the host cell membrane and thus inhibits virus release (Neil et al., 2008; Van Damme et al., 2008).

Vpu-induced downregulation of CD4 is a multistep process that is initiated by physical binding of phosphorylated Vpu to CD4. The cytoplasmic domains of the two proteins have been implicated in this interaction (Bour et al., 1995; Margottin et al., 1996; Tiganos et al., 1997). Mutational studies indicated that certain regions in the 
cytoplasmic domain of CD4 are crucial for Vpu binding: residues $414-419$ as well as a putative $\alpha$-helical structure in the membrane-proximal part of the cytoplasmic CD4 domain have been pointed out (Lenburg and Landau, 1993; Vincent et al., 1993; Willey et al., 1994; Yao et al., 1995).

Margottin et al. (1996) used a $\mathrm{Vpu}(28-81)$ peptide and the cytoplasmic domain of CD4 in a yeast two-hybrid assay to explore the CD4-Vpu interaction. They observed that residues 28-47 and 76-81 of Vpu are involved in CD4 binding, which are located in the cytoplasmic domain of Vpu. In the same report it was shown that CD4 degradation is abandoned if a Vpu double mutant lacking the two phosphorylation sites ( $552 \mathrm{~N}$ and S56N) is used in the assay. Tiganos et al. (1997) coexpressed full-length Vpu and the cytoplasmic domain of CD4 in African green monkey kidney cells. This study indicated that residues 30-50 in the cytoplasmic domain of Vpu are involved in the interaction with CD4.

Recent data suggest that residues in the TM domains of both Vpu and CD4 may also play an active role in the CD4-Vpu interaction (Magadan et al., 2010; Magadan and Bonifacino, 2012).

Studies with full-length Vpu and a CD4 fragment containing the TM and cytoplasmic domains, which were overexpressed in HeLa cells, indicated that two residues in the TM domain of Vpu, Val20, and Ser23, as well as Gly390 in the TM domain of CD4, are involved in the interaction between CD4 and Vpu (Magadan and Bonifacino, 2012).

NMR spectroscopy may reveal structural information on a residue-specific level. Both $\mathrm{Vpu}(39-81)$, reflecting the C-terminal part of the cytoplasmic domain of $\mathrm{Vpu}$ (Wittlich et al., 2009) as well as a CD4 fragment comprising the TM and the cytoplasmic domains of CD4, referred to as CD4(372-433) in what follows (Wittlich et al., 2007a, 2010), were characterized by solution NMR spectroscopy in the presence of detergent micelles. Further, the interaction between the cytoplasmic domain of $\mathrm{Vpu}$ and CD4(372-433) in micelles was analyzed by chemical shift perturbation and paramagnetic relaxation enhancement measurements (Singh et al., 2012). It was found that both helices in the cytoplasmic domain of Vpu are affected by CD4 binding and that Vpu binds to a membrane proximal region in the cytoplasmic domain of CD4. However, the affinity of CD4(372-433) for $\mathrm{Vpu}(39-81)$ in the micellar environment was unexpectedly low (Singh et al., 2012). A more realistic study of the CD4-Vpu interaction should be possible if both interaction partners (i) contain at least the TM and the cytoplasmic domain and (ii) are reconstituted in the same lipid bilayer membrane. The resulting proteoliposomes are too large for solution-state NMR studies. Solid-state NMR spectroscopy offers a viable alternative for investigations of membrane proteins reconstituted in native phospholipid bilayers (McDermott, 2009; Marassi et al., 2011; Yang et al., 2011; Hong et al., 2012; Miao et al., 2012).

The structure, dynamics, and oligomerization of the TM domain of Vpu in lipid bilayers have been studied extensively with solid-state NMR spectroscopy (Sharpe et al., 2006; Park and Opella, 2007; Lu et al., 2010). Opella and coworkers determined the tilt and rotation angle of the TM helix of Vpu in mechanically aligned phospholipid bilayers (Park et al., 2003; Park and Opella, 2005) and magnetically aligned phospholipid bicelles (Park et al., 2006a) of different hydrophobic thicknesses. Preliminary solid-state NMR spectra have been published by the Opella lab using chemically synthesized (Kochendoerfer et al., 2004) and recombinantly produced full-length Vpu (Cook et al., 2011).

Here we report the reconstitution of recombinantly expressed and uniformly ${ }^{13} \mathrm{C}$ - and ${ }^{15} \mathrm{~N}$-labeled full-length Vpu and CD4(372-433) into 1-palmitoyl-2-oleoyl-sn-glycero-3-phosphocholine (POPC) bilayers and their initial characterization by magic angle spinning (MAS) solidstate NMR spectroscopy.

\section{Results and discussion}

\section{CD4(372-433) shows higher mobility in POPC bilayers than full-length $\mathrm{Vpu}$}

Either of the two uniformly ${ }^{13} \mathrm{C}$ - and ${ }^{15} \mathrm{~N}$-labeled proteins CD4(372-433) and full-length Vpu was reconstituted into POPC bilayers. The resulting proteoliposomes were lyophilized, packed into sample containers for MAS, and rehydrated moderately prior to the MAS solid-state NMR measurements.

The temperature of the gel-to-liquid crystalline-phase transition of POPC in the proteoliposomes was estimated based on series of $1 \mathrm{D}{ }^{1} \mathrm{H}$ MAS spectra recorded with a temperature increment of $5 \mathrm{~K}$. The samples contained $\sim 12$ molecules of water per POPC and the molar lipid-toprotein ratio was $\sim 87$ in the case of Vpu or $\sim 73$ in the case of CD4(372-433). ${ }^{1} \mathrm{H}$ MAS spectra are dominated by the lipid and water signals, and pronounced line broadening is expected below the main-phase transition temperature (cf. Figure S1). The lipid phase transition occurred between $-5^{\circ} \mathrm{C}$ and $0^{\circ} \mathrm{C}$ in both proteoliposome preparations. This agrees with the previously reported phase-transition 
temperature of $-2.5^{\circ} \mathrm{C}$ for fully hydrated protein-free POPC liposomes (Litman et al., 1991).

In order to probe the mobility of the reconstituted proteins within the liposomes, $1 \mathrm{D}^{13} \mathrm{C}$ MAS spectra of both samples were recorded using different mechanisms for polarization transfer from ${ }^{1} \mathrm{H}$ to ${ }^{13} \mathrm{C}$ spins. Dipolar coupling-based CP (Hartmann and Hahn, 1962; Pines et al., 1973) is effective if the vector connecting the interacting nuclei is restricted in mobility and correlation times of residual motions are above the millisecond range. In contrast, ${ }^{1} \mathrm{H}-{ }^{13} \mathrm{C}$ polarization transfer by insensitive nuclei enhanced by polarization transfer (INEPT) (Morris and Freeman, 1979; Burum and Ernst, 1980) is effective only if dipolar couplings are averaged out by large amplitude motions on time scales of $<10^{-5} \mathrm{~s}$ (Andronesi et al., 2005; Yang et al., 2011). Slower motions in the range between milliseconds and microseconds on the other hand can affect $T_{1 \rho}$ relaxation and thus decrease the CP efficiency even if the amplitude of the motion is not large enough for isotropic averaging of the dipolar coupling (Fares et al., 2005; Sharpe et al., 2006).

CP-based ${ }^{13} \mathrm{C}$ MAS spectra of proteoliposomes containing either $\mathrm{CD} 4(372-433)$ or $\mathrm{Vpu}$ show ${ }^{13} \mathrm{C}$ signals typical for proteins both above and below the lipid phase transition temperature (Figure 1). However, INEPT-based spectra display only signals from POPC acyl chains and head groups but no protein signals. This remains true even above the lipid phase transition (Figure S3). These observations indicate limited mobility of both proteins in the lipid membrane and at least partial immobilization of protein regions on the millisecond time scale at the given hydration level.

The intensity of all CP-based signals of CD4(372433) increases significantly upon decreasing the temperature below the POPC gel-to-liquid crystalline-phase transition (Figure 1A, blue). However, the intensity of CP-based Vpu signals is already high in the liquid crystalline phase and increases only slightly in the lipid gel phase. This significantly different temperature dependence of signal intensities of CD4(372-433) and Vpu was reproduced in double-quantum (DQ) SPC-5 spectra recorded at $-20^{\circ} \mathrm{C}$ and $8^{\circ} \mathrm{C}$ (Figure S5.A). Signals from Gly, Ile, Val, Phe, and Leu residues, which constitute the major fraction of amino acids of the TM domain of CD4(372-433) (Figure S8), are significantly reduced at $8^{\circ} \mathrm{C}$ (Figure S5.A, red). Further, $\mathrm{C} \alpha \mathrm{C} \beta$ cross-correlations from amino acids Glu, Gln, Lys, His, Arg, and Thr, which are predominantly found in the cytoplasmic domain of CD4, are completely missing at $8^{\circ} \mathrm{C}$. Upon lowering the temperature from $8^{\circ} \mathrm{C}$ to $-20^{\circ} \mathrm{C}$, the peak volumes of CD4(372-433) signals from hydrophobic residues dominating the TM domain increase by a factor between 5 and 13, while those from polar residues increase by a factor of 15 or more. In contrast, the DQ correlation (DQC) spectra of Vpu display strong cross-correlations not only for amino acids from the TM helix, but from all domains of Vpu even at temperatures above the lipid phase transition (Figure S5.B). Decreasing the temperature to $-20^{\circ} \mathrm{C}$ causes only little further signal enhancement by a factor between 1.1 and 1.7 .
A

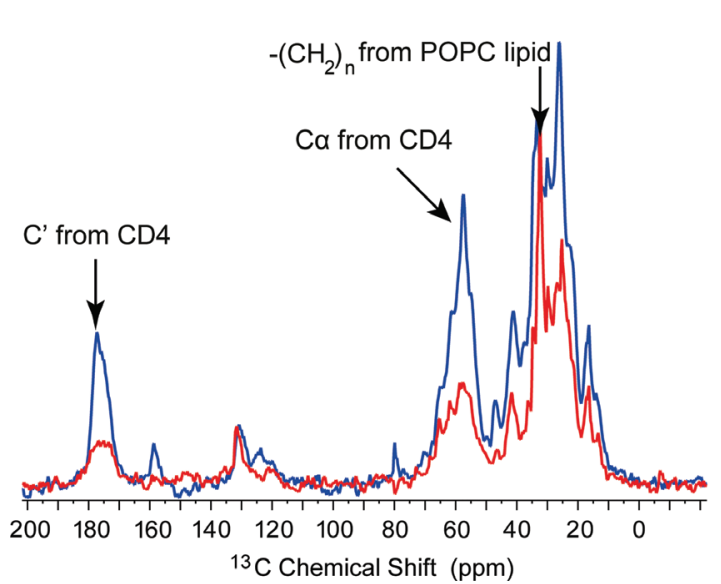

B

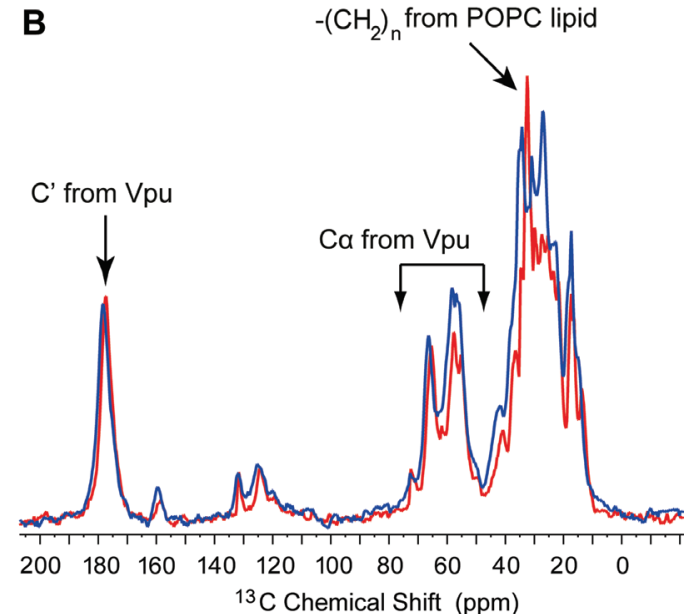

Figure $1{ }^{13} \mathrm{C}$ CP MAS spectra CP signals of uniformly labeled ${ }^{13} \mathrm{C},{ }^{15} \mathrm{~N} C D 4(372-433)(\mathrm{A})$ and full-length Vpu protein (B) in POPC bilayers. The experiments were conducted for $\mathrm{CD} 4(372-433)$ at temperatures of $5^{\circ} \mathrm{C}$ (red) and $-20^{\circ} \mathrm{C}$ (blue) and for Vpu at temperatures of $8^{\circ} \mathrm{C}($ red) and $-20^{\circ} \mathrm{C}$ (blue). The spectra were recorded at a field strength of $14.1 \mathrm{~T}$ with a spinning speed of $8.0 \mathrm{kHz}, 16 \mathrm{scans}$ each. All spectra were processed with an exponential line broadening of $20 \mathrm{~Hz}$. 
These results suggest that the cytoplasmic domain of CD4(372-433) in hydrated liposomes above the lipid phase transition temperature has a substantial degree of flexibility despite the rather low hydration level. However, the molecular fluctuations are either too slow or occur with an amplitude too small for isotropic averaging of dipolar interactions on the NMR time scale $\left(<10^{-5} \mathrm{~s}\right)$. Likewise, signals from the TM domain seem to be highly affected by mobility of the sample. For Vpu, in contrast, signals from residues of both domains are affected to a much lesser degree by slow motions. As already demonstrated in 1975 (Cherry, 1975), membrane proteins in lipid bilayers may undergo fast rotational reorientation about the bilayer normal, which leads to a reduction of anisotropic interactions by a factor of $0.5\left(3 \cos ^{2} \varphi-1\right)$, where $\varphi$ is the tilt angle of the long axis of the TM helix toward the bilayer normal (Lewis et al., 1985). For the TM domain of $\mathrm{Vpu}$, it could be demonstrated for various bilayer systems that the TM domain undergoes fast rotation about the bilayer normal, but not around the long axis of the helix (De Angelis et al., 2004; Park et al., 2006b). In the same set of studies, the Vpu TM domain has been shown to form at least transiently homo-oligomers, thus restricting the protein mobility at a given hydration level (Lu et al., 2010). However, in the case of CD4(372-433), additional motions of the TM helix within the bilayer may be possible. Further, the larger cytoplasmic domain of full-length $\mathrm{Vpu}$ may show stronger interactions with the bilayer surface than the TM domain of Vpu alone, which perhaps restricts domain mobility and thus leads to a reduced amplitude of slow motions. To further shed light on the dynamics of both membrane proteins, we recorded static ${ }^{13} \mathrm{C}$ spectra of both compounds (Figure S4). Although the powder patterns of carbonyl ${ }^{13} \mathrm{C}$ atoms, $\mathrm{C} \alpha$, and aliphatic side chain resonances are heavily overlapped, some conclusions can be drawn from the sum of the powder patterns of the carboxyl carbon atoms. For $\mathrm{Vpu}$, the powder pattern spans an area from roughly $250 \mathrm{ppm}$ to below $100 \mathrm{ppm}$, with a maximum at about $195 \mathrm{ppm}$. These values are in close agreement with experimentally determined chemical shift anisotropy (CSA) powder patterns obtained on completely immobilized model peptides (Wei et al., 2001). Thus, our data suggest that for Vpu at the low hydration level, rotational averaging is to a large degree suppressed. For CD4(372433) the combined CSA powder pattern is much less resolved but clearly seems reduced with respect to the full powder pattern, and the same trend can be observed for the side-chain carbon atoms. These findings support the suspicion that CD4(372-433) in the given sample undergoes some rotational averaging of anisotropic interactions. Likewise, $T_{10}$ values for ${ }^{1} \mathrm{H}$ and ${ }^{13} \mathrm{C}$ were significantly reduced for CD4(372-433) in comparison to Vpu (Table S2).

Taken together, our data show that CD4(372-433) in POPC bilayers above the lipid phase transition temperature has a higher degree of flexibility than Vpu.

\section{Resonance assignment of CD4(372-433) and Vpu protein in POPC bilayers}

The first step toward structural information is residue-specific resonance assignment. Toward this goal, we recorded ${ }_{2 \mathrm{D}}{ }^{13} \mathrm{C} /{ }^{13} \mathrm{C}$ and ${ }^{13} \mathrm{C} /{ }^{15} \mathrm{~N}$ correlation spectra of uniformly ${ }^{13} \mathrm{C}$ and ${ }^{15} \mathrm{~N}$-labeled CD4(372-433) and Vpu. Although the line widths of 1-5 ppm, the unfavorable distribution of amino acid types, and the low dispersion of chemical shifts prohibited a complete site-specific resonance assignment in both cases, 13 assignments for residues from the TM domain of CD4(372-433) and 2 site-specific assignments for Vpu could be obtained. Representative assignments of CD4 and Vpu are shown in Figures 2 and 3, respectively.

Figure 2 represents the overlay of two $2 \mathrm{D}{ }^{13} \mathrm{C} /{ }^{13} \mathrm{C}$ correlation spectra obtained with short ( $20 \mathrm{~ms}$, black) and long (200 ms, gray) mixing times for proton-driven spin diffusion (PDSD). Eight amino acid types, i.e., Ala, Val, Ile, Leu, Pro, Phe, Gly, and Ser, were identified via intraresidue cross peaks at the short mixing time (black spectrum), whereas the spin system of Arg could be identified in the spectrum recorded with a longer PDSD mixing time. The spinning speed was deliberately set to $12.5 \mathrm{kHz}$, which is close to the $n=2$ rotational resonance condition between $\mathrm{CO}$ and $\mathrm{C} \alpha$ resonances. Thus, longitudinal magnetization exchange between $\mathrm{CO}$ and $\mathrm{C} \alpha$ of neighboring residues via the backbone is particularly favored for longer mixing times, and subsequent relay transfer to all other carbon atoms of the side chain gives rise to interresidual cross peaks mainly between residues adjacent to each other in the primary sequence (Seidel et al., 2004). For CD4, interresidue cross peaks (gray spectrum) were found between residue pairs such as Leu/Val, Leu/Ile, Leu/Phe, Ile/Gly, Gly/Val, Ala/Leu, Gly/Leu, and Val/Ala. Remarkably, pairs of neighboring Ala/Leu, Leu/Ile, Gly/Val, Val/Ala, and Leu/Phe occur only once in the amino acid sequence of CD4(372-433) (Figure S8). Consequently, the interresidue cross peaks were specifically assigned to amino acid pairs Ala373/Leu374, Leu374/Ile375, Gly379/Val380, Val380/ Ala381, and Leu385/Phe386. In addition, interresidue cross peaks in the $2 \mathrm{D}{ }^{15} \mathrm{~N} /{ }^{13} \mathrm{C}$ correlation spectrum (Figure S9) could be identified for the unique pairs Gly378/Gly379 and Ile387/Gly388. Difference secondary chemical shift 


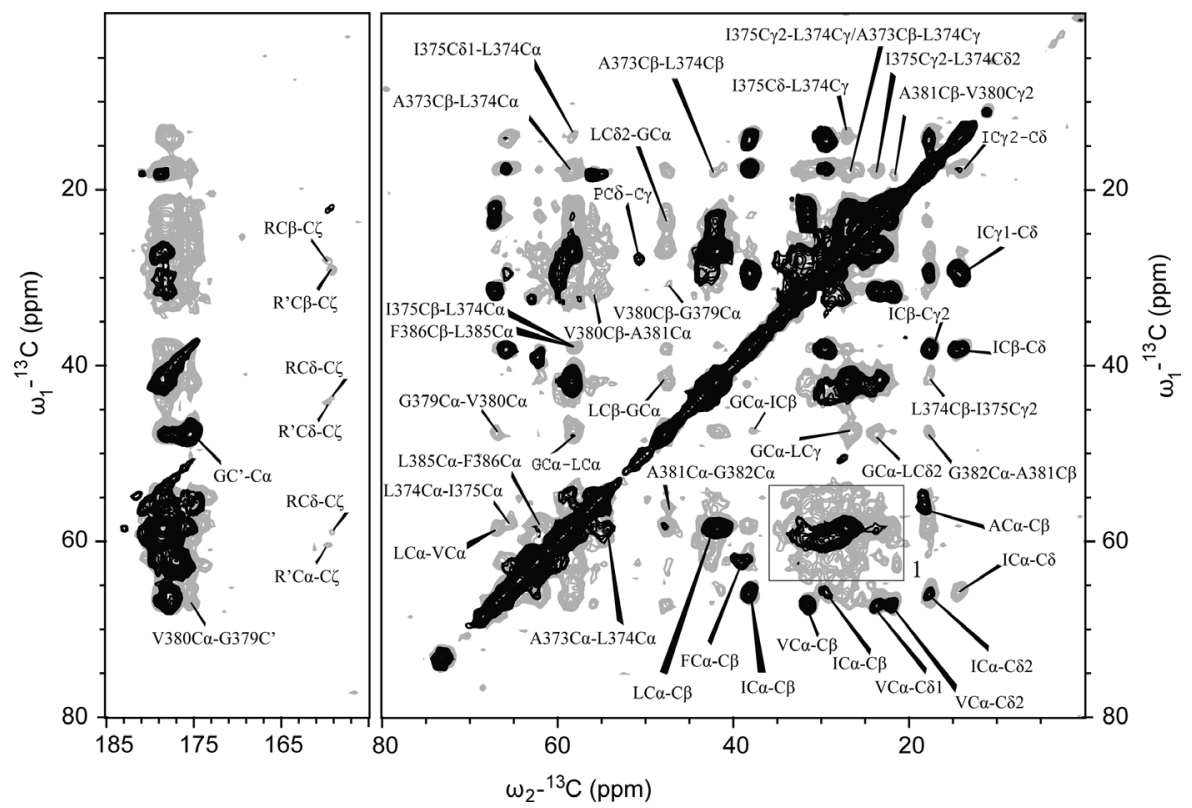

Figure $22 \mathrm{D}{ }^{13} \mathrm{C} /{ }^{13} \mathrm{C}$ correlation PDSD spectra of uniformly ${ }^{13} \mathrm{C},{ }^{15} \mathrm{~N}$ labeled CD4 (372-433) in POPC bilayers.

The spectra were acquired at a field strength of $18.8 \mathrm{~T}$ with a spinning speed of $12.5 \mathrm{kHz}$. The ${ }^{13} \mathrm{C}$ mixing times are $20 \mathrm{~ms}$ (black) and $200 \mathrm{~ms}$ (gray). The experiments were conducted at temperatures of $0^{\circ} \mathrm{C}$ and $\sim-5^{\circ} \mathrm{C}$ for the black and gray spectra, respectively. The data were processed using Lorentzian-to-Gaussian apodization with $100 \mathrm{~Hz}$ of Lorentzian line sharpening and $200 \mathrm{~Hz}$ of Gaussian line broadening prior to Fourier transformation.

values $\Delta \delta$ (Luca et al., 2001) were derived from $\mathrm{C} \alpha$ and $\mathrm{C} \beta$ chemical shifts of these specifically identified residues, Ala373, Leu374, Ile375, Gly378, Gly379, Val380, Ala381,
Gly382, Leu385, and Phe386, and turned out to be greater than $1.5 \mathrm{ppm}$, indicative of $\alpha$-helical secondary structure (Figure S10).

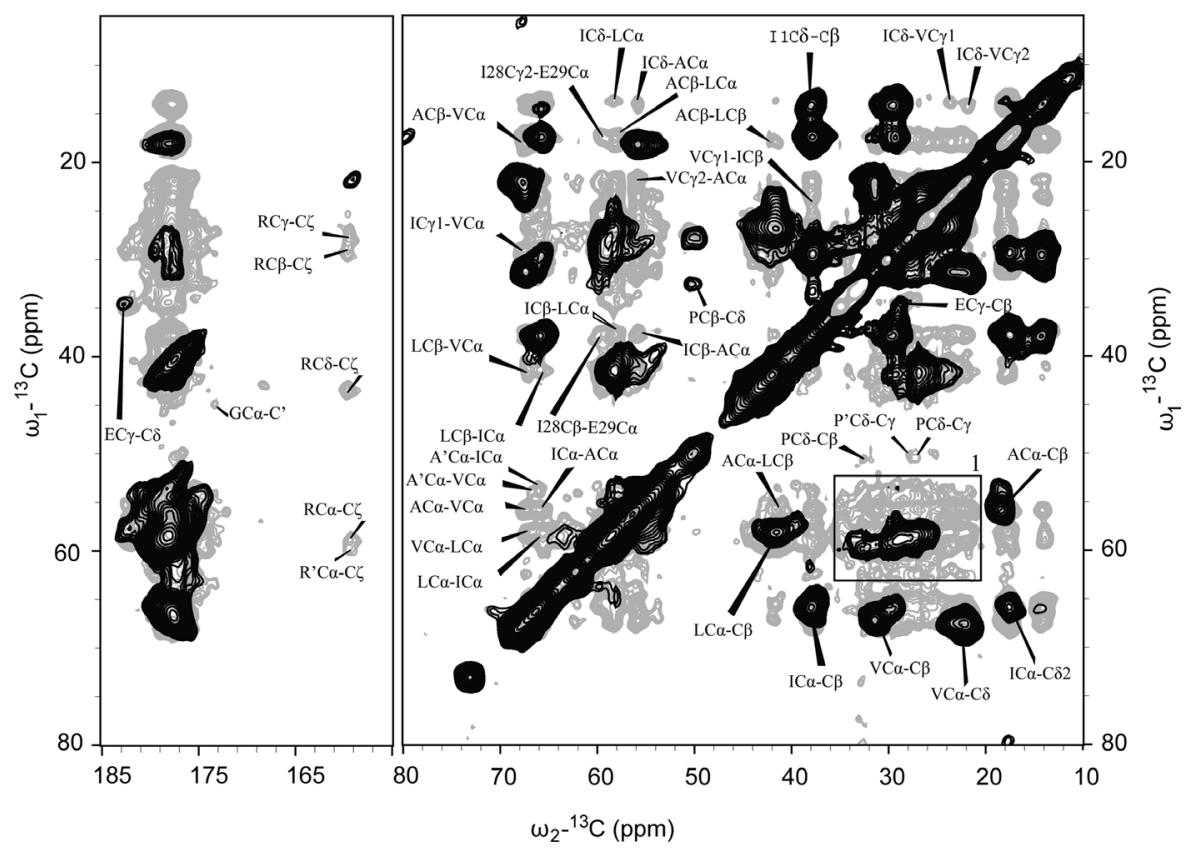

Figure $32 \mathrm{D}^{13} \mathrm{C} /{ }^{13} \mathrm{C}$ correlation PDSD-RR spectra of the uniformly labeled ${ }^{13} \mathrm{C},{ }^{15} \mathrm{~N}$ full-length Vpu protein in POPC bilayers.

The spectra were acquired at field strengths of $14.1 \mathrm{~T}$ (black) and $18.8 \mathrm{~T}$ (gray) with a spinning speed of 9.375 and $12.5 \mathrm{kHz}$, respectively. The ${ }^{13} \mathrm{C}$ mixing times are $10 \mathrm{~ms}$ (black) and $300 \mathrm{~ms}$ (gray). The experiment was conducted at a temperature of $0^{\circ} \mathrm{C}$. Data were processed using Lorentzian-to-Gaussian apodization with $100 \mathrm{~Hz}$ of Lorentzian line sharpening and $200 \mathrm{~Hz}$ of Gaussian line broadening prior to Fourier transformation. 
For the polar residues Glu, Gln, Lys, His, and Arg, which predominantly reside in the cytoplasmic domain of CD4(372-433), no amino acid type-specific resonance assignment could be obtained due to the extensive signal overlap of cross-correlation signals for these residues (indicated by a box in Figure 2). However, comparison of a DQC spectrum of CD4(372-433) in liposomes obtained at $-30^{\circ} \mathrm{C}$ with carbon chemical shifts from solution NMR data of CD4(372-433) in detergent micelles (Wittlich et al., 2007a, 2010) indicates that (i) all signals from the cytoplasmic part of CD4(372-433) in POPC bilayers can be observed at least at low temperatures in solid-state NMR spectra, (ii) no major shift changes between both sample preparations is observed, and (iii) the resolution for residues of the cytoplasmic domain is quite low in the frozen sample, which may be indicative of a high degree of conformational disorder in the frozen state.

For Vpu in POPC bilayers, two $2 \mathrm{D}{ }^{13} \mathrm{C} /{ }^{13} \mathrm{C}$ correlation spectra recorded with a short (10 ms, black) and long (300 ms, gray) PDSD mixing times are shown (Figure 3). The line widths of the overlapping cross peaks in Figure 3 are on the order of 1-5 ppm. Due to the rather large line widths, site-selective resonance assignments were not possible, except for the unique pair I28/E29. From the intraresidue cross peaks in the spectrum with short mixing time, spin systems for six amino acid types (Ala, Val, Ile, Leu, Pro, and Glu) could be identified. Interresidue cross peaks were observed for the pairs Ala/Leu, Ala/Ile, Ala/Val, Leu/Val, Ile/Leu, and Ile/Val. Each of the residue types Ala, Val, and Ile occurs five times in the 18-residue Vpu TM helix, and sequential pairs of these three residues are exclusively found in the Vpu TM domain. This allowed us to identify two Ala, one Ile, and one Val spin system which must reflect amino acids in the TM region of Vpu. Corresponding ${ }^{13} \mathrm{C}$ chemical shifts were deduced from the well-resolved interresidue cross-correlations. The secondary chemical shift parameter $\Delta \delta$ was calculated for these four residues from their $\mathrm{C} \alpha$ and $\mathrm{C} \beta$ chemical shifts and all four values are highly positive ( $>1.5 \mathrm{ppm})$. This is in agreement with an $\alpha$-helical conformation of the TM domain of $\mathrm{Vpu}$ in POPC bilayers.

$\mathrm{C} \alpha / \mathrm{C} \beta$ cross-correlations of the polar Vpu residues Glu, Gln, Arg, His, Trp, and Lys are present in the spectra (box in Figure 3) but are not resolved. ${ }^{13} \mathrm{C}$ carbon shifts of the cytoplasmic domain of $\mathrm{Vpu}$ in the presence of detergent micelles are available from liquid-state NMR (Wittlich et al., 2009). They reasonably well match many ${ }^{13} \mathrm{C} /{ }^{13} \mathrm{C}$ correlations of $\mathrm{Vpu}$ in liposomes detected in the DQ correlation spectrum (DQS). This is particularly true for liquid-state NMR ${ }^{13} \mathrm{C}$ shifts of residues Glu48, Arg49, Glu51, Glu56, Glu58, Gln61, Glu62, Glu63, Glu69, Arg70,
His72, and Trp76. These observations suggest a similar secondary structure of the cytoplasmic domain of Vpu in the presence of micelles and in proteoliposomes.

Residue-specific assignments of ${ }^{13} \mathrm{C}$ chemical shifts of CD4 and Vpu in liposomes are listed in Tables S3 and S5, respectively. Additional amino acid type-specific assignments are summarized in Tables S4 and S6, respectively.

\section{Topology of CD4(372-433) and Vpu in POPC bilayers}

In order to check if the TM domains were correctly inserted into the lipid bilayer, we probed the topology of CD4(372433) and Vpu in POPC bilayers by $2 \mathrm{D}$ and $3 \mathrm{D}{ }^{1} \mathrm{H},{ }^{1} \mathrm{H}$ spin diffusion experiments (Kumashiro et al., 1998; Huster et al., 2002). Proton magnetization from mobile fractions of the sample (i.e., $\mathrm{H}_{2} \mathrm{O}$ and POPC acyl chains) is selected by a $\mathrm{T}_{2}$ relaxation filter (Kumashiro et al., 1998) and, after an evolution period, transferred to the protein via ${ }^{1} \mathrm{H}$ spin diffusion and finally $\mathrm{CP}$ to ${ }^{13} \mathrm{C}$. Thus, a $1 \mathrm{D}{ }^{13} \mathrm{C}$ spectrum is correlated with each ${ }^{1} \mathrm{H}$ chemical shift of surrounding water or acyl side chains. 3D HCC spectra correlating the ${ }^{1} \mathrm{H}$ chemical shift of the magnetization source with a $2 \mathrm{D}$ ${ }^{13} \mathrm{C} /{ }^{13} \mathrm{C}$ correlation spectrum can be obtained by combining a proton dimension via PDSD with a $2 \mathrm{D}{ }^{13} \mathrm{C} /{ }^{13} \mathrm{C}$ correlation spectrum (Kijac et al., 2010). As a result, subspectra of the protein are observed that are correlated with a specific ${ }^{1} \mathrm{H}$ chemical shift (e.g., of water protons at $\sim 4.9 \mathrm{ppm}$ or of $\mathrm{CH}_{2}$ protons at $\sim 1.2 \mathrm{ppm}$ ). Importantly, the ${ }^{1} \mathrm{H}$ nuclei giving rise to the ${ }^{1} \mathrm{H}$ signal and the ${ }^{13} \mathrm{C}$ nuclei of the protein observed in the ${ }^{13} \mathrm{C}$ dimension(s) must be in close proximity for efficient polarization transfer. Thus, protein domains inserted into the lipid bilayer can be easily distinguished from water-accessible protein domains.

The optimal proton spin diffusion time was determined from magnetization build-up curves for different correlations in 2D experiments (Figure S12). In all cases, the build-up curves reached a plateau after $\sim 10 \mathrm{~ms}$. To probe the topology of the Vpu and CD4(372-433), we chose proton spin diffusion times of 2-3 ms.

\section{The TM domain of CD4(372-433) is inserted correctly in POPC bilayers}

The high mobility of CD4 in proteoliposomes with a waterto-lipid ratio of 12:1 leads to very poor intensities in wateredited spectra acquired at a temperature above the lipid phase transition. Therefore, we used a different sample with a water-to-lipid ratio of $\sim 6: 1$, in which the protein 
signals in $\mathrm{CP}$ and $\mathrm{DQ}$ spectra are significantly stronger due to reduced mobility of $\mathrm{CD} 4(372-433)$ to probe the topology of CD4(372-433) in POPC bilayers. Figure 4 shows a 2D spectrum correlating the ${ }^{1} \mathrm{H}$ signals of the water and the POPC acyl chains, respectively, with subsets of ${ }^{13} \mathrm{C}$ signals of the protein. Comparison with a ${ }^{1} \mathrm{H},{ }^{13} \mathrm{C}$ HETCOR spectrum of POPC liposomes (red spectrum in Figure 4) indicates that most ${ }^{13} \mathrm{C}$ signals that are correlated with ${ }^{1} \mathrm{H}$ resonances of the lipid acyl chains (blue spectrum) do not match ${ }^{13} \mathrm{C}$ signals of POPC and thus must originate from CD4(372-433). Likewise, for the ${ }^{13} \mathrm{C}$ signals correlated with the ${ }^{1} \mathrm{H}$ chemical shift of water, at least some resonances in the $\mathrm{C} \alpha$ region can be identified that are not overlapping with lipid ${ }^{13} \mathrm{C}$ resonances.

The ${ }^{13} \mathrm{C}$ signals correlated with protons from the POPC acyl chains are reasonably well resolved, and chemical shifts can be assigned to the hydrophobic residues Ile, Val, Leu, and Ala, which constitute the major part of the TM domain of $\mathrm{CD} 4$. Furthermore, one cross peak at a ${ }^{13} \mathrm{C}$ chemical shift in the range of 46-49 ppm, which matches the $\mathrm{C} \alpha$ chemical shifts of G378, G379, and G382, is identified only in the correlation with protons from the lipid bilayer, but not with water protons. As these three glycine residues are located in the TM helix of CD4(372-433), the 2D correlation suggests that the hydrophobic TM helix is inserted into the lipid bilayer and well shielded from the water.

In contrast, the ${ }^{13} \mathrm{C}$ trace correlated with water protons appears much less resolved, showing peaks that are in agreement with $\mathrm{C} \alpha$ random coil chemical shifts of the polar residues Glu, Gln, Lys, His, Trp, and Arg, which constitute the major fraction of the cytoplasmic domain. The rather broad and unresolved signals are also in agreement with a rather unstructured and flexible conformation of the cytoplasmic domain, as observed in the $2 \mathrm{D}{ }^{13} \mathrm{C} /{ }^{13} \mathrm{C}$ correlation spectra.

$3 \mathrm{D}{ }^{1} \mathrm{H} /{ }^{13} \mathrm{C} /{ }^{13} \mathrm{C}$ correlation spectra of $\mathrm{CD} 4(372-433)$ in POPC (Figure S13, C and D) confirm these findings: Cross peaks characteristic for the hydrophobic residues Val, Ile and Leu were identified in the ${ }^{13} \mathrm{C} /{ }^{13} \mathrm{C}$ plane of a 3D spectrum at the ${ }^{1} \mathrm{H}$ chemical shift of the POPC acyl chains, whereas the ${ }^{13} \mathrm{C} /{ }^{13} \mathrm{C}$ plane correlated with the ${ }^{1} \mathrm{H}$ chemical shift of water lacks well-resolved cross peaks.

\section{The TM domain of Vpu inserts into the POPC bilayer, whereas the cytoplasmic domain is exposed to water}

In Figure 5, two planes of a 3D HCC correlation spectrum of $\mathrm{Vpu}$ are shown, which display $2 \mathrm{D}{ }^{13} \mathrm{C} /{ }^{13} \mathrm{C}$ correlation spectra correlated with the ${ }^{1} \mathrm{H}$ chemical shifts of water (red) and the POPC acyl chains (black), respectively. The spectrum was recorded above the lipid phase transition temperature, the ${ }^{1} \mathrm{H},{ }^{1} \mathrm{H}$ spin diffusion time was set to 3 $\mathrm{ms}$, which is well before the magnetization reaches equilibrium throughout the sample. The 2D plane correlated with the ${ }^{1} \mathrm{H}$ shift of the lipid hydrocarbon chain is dominated by pronounced intraresidue cross peaks of spin systems reflecting the hydrophobic residues Ala, lle, and Val. In addition, some interresidue cross peaks such as AlaC $\alpha$-ValC $\alpha$ and IleC $\alpha$-AlaC $\alpha$ could be observed. The TM domain of Vpu consists of 18 residues; the most abundant amino acids within this TM helix are Val (5), Ile (5), and Ala (5). Furthermore, two Ala/Val pairs and four Ala/Ile pairs, which may give rise to the interresidue cross peaks, are found exclusively in the TM helix (Figure S11). The

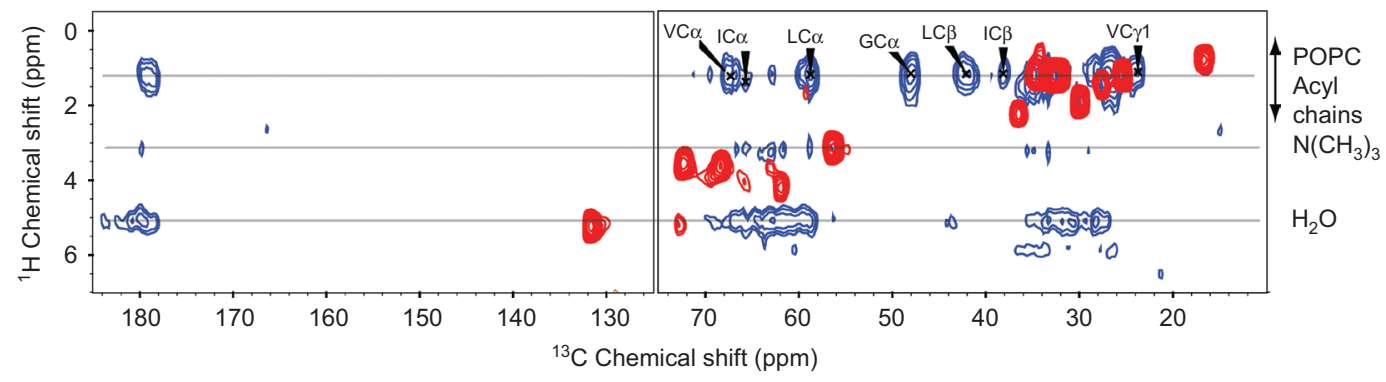

Figure 4 Overlay of $2 \mathrm{D}^{1} \mathrm{H} /{ }^{13} \mathrm{C}$ spectra of the uniformly ${ }^{13} \mathrm{C},{ }^{15} \mathrm{~N}$ labeled $\mathrm{CD} 4(372-433)$ (blue) in POPC lipid bilayers and of the unlabeled POPC lipid (red).

The water-to-lipid ratio is roughly 6:1. The blue spectrum was recorded with a $T_{2}$ before and ${ }^{1} \mathrm{H}$ spin diffusion after the indirect proton evolution period to separate water-accessible and lipid-embedded regions of the protein. The $T_{2}$ filter time was set to $100 \mu \mathrm{s}$, and the longitudinal mixing time for proton spin diffusion was $2 \mathrm{~ms}$. The red HETCOR spectrum was recorded using INEPT transfer from protons to carbons (128 scans). The experiments were conducted at temperatures of $0^{\circ} \mathrm{C}$ (blue) and $8^{\circ} \mathrm{C}$ (red), respectively, at a field strength of $14.1 \mathrm{~T}$ with a spinning speed of $9.375 \mathrm{kHz}$. Data were processed using a cosine-squared bell apodization prior to Fourier transformation. Carbon signals (blue) correlated with ${ }^{1} \mathrm{H}$ chemical shifts from protons of the POPC acyl chain and $\mathrm{H}_{2} \mathrm{O}$ belong to protein parts embedded in the lipid bilayer or accessible to free water, respectively. 


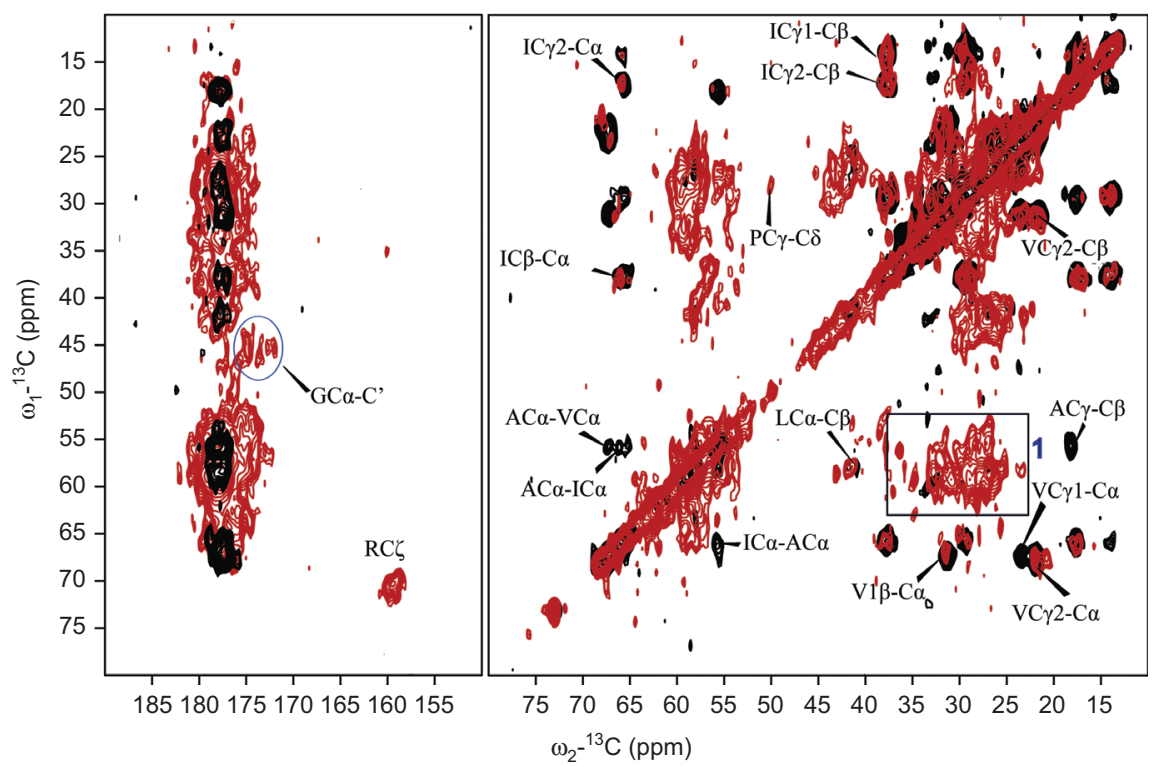

Figure 5 Overlay of $2 \mathrm{D}^{13} \mathrm{C} /{ }^{13} \mathrm{C}$ planes from $3 \mathrm{D}^{11} \mathrm{H} /{ }^{13} \mathrm{C} /{ }^{13} \mathrm{C}$ spectrum recorded with a $T_{2}$ filter and ${ }^{1} \mathrm{H}$ spin diffusion of liposome-reconstituted Vpu.

The planes display 2D carbon correlation spectra from protein parts close to free water (red) and lipid $\mathrm{CH}_{2}$ protons (black). The experiment was conducted at a temperature of $0^{\circ} \mathrm{C}$ (above the temperature of the POPC gel to liquid phase transition). The spectrum was acquired at a field strength of $18.8 \mathrm{~T}$ with a spinning speed of $12.5 \mathrm{kHz}$ and 96 scans. The $T_{2}$ filter time was $240 \mu \mathrm{s}$, the ${ }^{1} \mathrm{H}$ spin diffusion was $3 \mathrm{~ms}$, and the ${ }^{13} \mathrm{C}$ mixing time was $50 \mathrm{~ms}$. Evolution times in the indirect ${ }^{1} \mathrm{H}$ and ${ }^{13} \mathrm{C}$ dimensions were 1.7 and $3.2 \mathrm{~ms}$, respectively. The data were processed with forward linear prediction for the ${ }^{1} \mathrm{H}$ dimension and cosine-squared bell apodization prior to Fourier transformation.

dominance of correlations from these three hydrophobic amino acids, in concert with the absence of clear indications of correlations reflecting polar amino acids in the ${ }^{13} \mathrm{C} /{ }^{13} \mathrm{C}$ plane that is correlated to the POPC acyl chain ${ }^{1} \mathrm{H}$ chemical shift, suggests that the TM domain of $\mathrm{Vpu}$ is correctly reconstituted into the hydrophobic core of the POPC bilayer, whereas the cytoplasmic domain of $\mathrm{Vpu}$ is excluded from the bilayer interior.

In the 2D plane correlated with the ${ }^{1} \mathrm{H}$ chemical shift of water, cross peaks characteristic for Gly, Pro, and Arg could be clearly identified (Figure 5). NMR signals of carbonyl carbons (170-185 ppm in the $\omega_{2}$ dimension) and the area where cross peaks from the polar amino acids Glu, Gln, Arg, His, Lys, and Trp are expected (boxed in Figure 5) are drastically enhanced in the plane correlated with the ${ }^{1} \mathrm{H}$ chemical shift of water. Amino acids of these types occur exclusively outside the TM domain of Vpu, i.e., either in the cytoplasmic or in the short extracellular domain preceding the TM helix (Figure S11). Our data reflect the water accessibility of the short $\mathrm{N}$-terminal and cytoplasmic domains of Vpu.

Taken together, these results suggest that the cytoplasmic part of $\mathrm{Vpu}$ in POPC bilayers is accessible to water, whereas the TM region is located in the hydrocarbon chain region. The broad line widths of the observed NMR signals may indicate conformational disorder.

\section{Conclusion}

Recombinantly produced full-length Vpu and CD4(372-433) were reconstituted into POPC bilayers, and the conformation, mobility, and topology of these proteins were studied on an atomic level. We found that (i) the CD4 protein reveals higher mobility than Vpu in POPC bilayers under comparable experimental conditions; (ii) in POPC lipid bilayers, the TM parts of CD4 and Vpu adopt an $\alpha$-helical structure; (iii) these TM domains are correctly inserted into the hydrophobic core of the lipid bilayers; and (iv) the cytoplasmic part of Vpu in POPC bilayers is in close proximity to water.

\section{Materials and methods}

\section{Sample preparation}

Uniformly ${ }^{13} \mathrm{C}$ - and ${ }^{15} \mathrm{~N}$-labeled $\mathrm{CD} 4(372-433)$ and full-length $\mathrm{Vpu}$ (HIV-1 strain HV1S1, Swiss-Prot accession number P19554) were expressed as ubiquitin fusion proteins in Escherichia coli C43(DE3) cells (BioCat $\mathrm{GmbH}$, Heidelberg, Germany) at $37^{\circ} \mathrm{C}$ in $\mathrm{M} 9$ medium containing ${ }^{13} \mathrm{C}$-glucose $(2 \mathrm{~g} / \mathrm{l})$ and ${ }^{15} \mathrm{~N}$-ammonium chloride $(1 \mathrm{~g} / \mathrm{l})$ (Eurisotop, Saarbrücken, Germany) as sole sources of carbon and nitrogen, respectively. The proteins were isolated and purified as described earlier by Wittlich et al. (2007b). 
Proteoliposomes were prepared from POPC (Avanti Polar Lipids, Alabaster, AL, USA) and uniformly ${ }^{13} \mathrm{C}$ - and ${ }^{15} \mathrm{~N}$-labeled protein [either CD4(372-433) or Vpu] by the organic solvent-mediated reconstitution method developed by Cross and coworkers (Page et al., 2007). First, for each NMR sample, $\sim 3 \mathrm{mg}$ of lyophilized protein was combined with $\sim 20 \mathrm{mg}$ POPC in an organic solvent mixture containing 30\% methanol and $70 \%$ chloroform. The lipid-to-protein molar ratio was estimated for each sample based on the individual amounts of POPC and protein used and was between 70:1 and 90:1. The organic solvent was removed by passing a stream of nitrogen gas over the sample in a glass test tube, leaving a dry lipid/protein film on the inner wall of the test tube. Second, the lipid/protein film was suspended in cyclohexane. The sample was frozen in liquid nitrogen and lyophilized overnight under high vacuum for complete removal of organic solvent. Third, fully hydrated proteoliposomes were obtained by suspending each POPC/protein sample $(-23 \mathrm{mg})$ in $490 \mu \mathrm{l}$ of $\mathrm{H}_{2} \mathrm{O}$ supplemented with $10 \mu \mathrm{l}$ of buffer (20 mM sodium phosphate, $\mathrm{pH} 7.4,70 \mathrm{~mm} \mathrm{NaCl}$, $0.02 \% \mathrm{NaN}_{3}$ ). Samples were subjected to five freeze/thaw cycles using liquid nitrogen and a water bath at room temperature to improve sample homogeneity. Finally, samples were frozen in liquid nitrogen and lyophilized for 8 to $16 \mathrm{~h}$, resulting in scarcely hydrated POPC/ protein mixtures. Each lyophilized lipid/protein sample was packed into a 3.2-mm rotor and between 2 and $5 \mu \mathrm{l}$ of $\mathrm{H}_{2} \mathrm{O}$ was added. Prior to NMR experiments, samples in the sealed rotors spinning inside the MAS probe were cycled five times between $-20^{\circ} \mathrm{C}$ and $+8^{\circ} \mathrm{C}$, i.e., temperatures below and above the main-phase transition of the lipid, respectively, in order to achieve sample homogeneity. The final waterto-lipid molecular ratio in each sample was derived from the integral intensities of the ${ }^{1} \mathrm{H}$ signals of $\mathrm{H}_{2} \mathrm{O}$ and of the choline methyl protons of POPC in $1 \mathrm{D}^{1} \mathrm{H}$ NMR spectra recorded under MAS conditions.

\section{MAS solid-state NMR experiments}

All experiments in the present work were carried out on Varian (Varian Inc., Palo Alto, CA, USA) VNMRS NMR spectrometers operating at field strengths of 14.1 and $18.8 \mathrm{~T}$ (corresponding to ${ }^{1} \mathrm{H}$ Larmor frequencies of 600 and $800 \mathrm{MHz}$ ), respectively. Triple-resonance $3.2 \mathrm{~mm}$ MAS probes tuned to either ${ }^{1} \mathrm{H} /{ }^{13} \mathrm{C}$ or ${ }^{1} \mathrm{H} /{ }^{13} \mathrm{C} /{ }^{15} \mathrm{~N}$ were used. Experiments were performed at sample temperatures from $-30^{\circ} \mathrm{C}$ to $+8^{\circ} \mathrm{C}$. Sample temperatures were indirectly determined with an accuracy of $\pm 5^{\circ} \mathrm{C}$ for each spinning speed using nickelocene as an external reference (Heise et al., 2001). Initial excitation was achieved by CP from protons to ${ }^{13} \mathrm{C}$ or ${ }^{15} \mathrm{~N}$, employing radio frequency (rf) fields between 51 and $60 \mathrm{kHz}$ on ${ }^{1} \mathrm{H}$, between 38 and $45 \mathrm{kHz}$ on ${ }^{13} \mathrm{C}$, and between
39 and $45 \mathrm{kHz}$ on ${ }^{15} \mathrm{~N}$ (Andrew et al., 1958; Lowe, 1959; Hartmann and Hahn, 1962; Pines et al., 1973). Proton decoupling using the small phase incremental alternation (SPINAL) decoupling scheme (Fung et al., 2000) employing rf field strengths from 80 to $90 \mathrm{kHz}$ was applied during acquisition and evolution periods. Proton spin diffusion experiments for determination of the topology (Kumashiro et al., 1998; Huster et al., 2002; Kijac et al., 2010) were acquired with $T_{2}$ filter times of 100 and $240 \mu$ s and proton mixing times of 2 to 3 ms. Refocused INEPT (Morris and Freeman, 1979; Burum and Ernst, 1980) was employed to probe rapidly tumbling protein domains. Static spectra were acquired with $\mathrm{CP}$ excitation and proton decoupling. ${ }^{1} \mathrm{H}$ and ${ }^{13} \mathrm{C} T_{10}$ measurements were performed at a spinning speed of $8 \mathrm{kHz}$ with ${ }^{13} \mathrm{C}$ detection, with a spin-lock pulse applied on ${ }^{1} \mathrm{H}$ before or on ${ }^{13} \mathrm{C}$ after $\mathrm{CP}$ transfer, respectively. Field strengths for the spin lock pulses were 30 $\mathrm{kHz}$ on the ${ }^{1} \mathrm{H}$ and $13 \mathrm{kHz}$ on the ${ }^{13} \mathrm{C}$ channel (with proton decoupling with a field strength of $76 \mathrm{kHz}$ ), respectively. For $2 \mathrm{D}^{13} \mathrm{C} /{ }^{13} \mathrm{C}$ correlation spectra, PDSD with mixing times from 20 to 300 ms (Bloembergen, 1949; Szeverenyi et al., 1982) was employed. DQ coherences for acquisition of DQS were excited and reconverted with the Supercycled POST-C5 (SPC5) sequence (Hohwy et al., 1999). In addition, interresidue correlations were obtained from PDSD spectra recorded close to the $\mathrm{CO}-\mathrm{C} \alpha$ rotational resonance (RR) condition, i.e., at rotor frequencies of $9.375 \mathrm{kHz}(14.1 \mathrm{~T})$ or $12.5 \mathrm{kHz}(18.8 \mathrm{~T})$, respectively, with mixing times of 100-300 ms (Seidel et al., 2004).

NMR data were processed with the NMRPipe software (Delaglio et al., 1995) using Lorentzian-to-Gaussian, sine-bell, or exponential apodization. ${ }^{13} \mathrm{C}$ chemical shifts were externally referenced using an adamantane sample. The upfield signal of adamantane was set to $31.4 \mathrm{ppm}$ on the DSS scale. ${ }^{1} \mathrm{H}$ and ${ }^{15} \mathrm{~N}$ chemical shifts were indirectly referenced based on ${ }^{13} \mathrm{C}$ shifts. Resonance assignments were performed using the Sparky software (Goddard and Kneller).

Acknowledgments: We thank Sameer Singh for useful discussions and help with sample preparation. We gratefully acknowledge financial support and training from the International NRW Research School BioStruct, granted by the Ministry of Innovation, Science and Research of the State North Rhine-Westphalia, the Heinrich Heine University of Düsseldorf, and the Entrepreneur Foundation at the Heinrich Heine University of Düsseldorf.

Received May 28, 2013; accepted July 13, 2013; previously published online July 16, 2013

\section{References}

Andrew, E.R., Bradbury, A., and Eades, R.G. (1958). Nuclear magnetic resonance spectra from a crystal rotated at high speed. Nature 182, 1659.

Andronesi, O.C., Becker, S., Seidel, K., Heise, H., Young, H.S., and Baldus, M. (2005). Determination of membrane protein structure and dynamics by magic-angle-spinning solid-state NMR spectroscopy. J. Am. Chem. Soc. 127, 12965-12974.

Bloembergen, N. (1949). On the interaction of nuclear spins in a crystalline lattice. Physica 15, 386-426.
Bour, S., Schubert, U., and Strebel, K. (1995). The human-immunodeficiency-virus type- 1 Vpu protein secifically binds to the cytoplasmic domain of CD4 - implications for the mechanism of degradation. J. Virol. 69, 1510-1520.

Burum, D.P. and Ernst, R.R. (1980). Net polarization transfer via a J-ordered state for signal enhancement of low-sensitivity nuclei. J. Magn. Reson. 39, 163-168.

Chengmayer, C., Quiroga, M., Tung, J.W., Dina, D., and Levy, J.A. (1990). Viral determinants of human-immunodeficiency-virus 
type-1 T-cell or macrophage tropism, cytopathogenicity, and CD4 antigen modulation. J. Virol. 64, 4390-4398.

Cherry, R.J. (1975). Protein mobility in membranes. FEBS Lett. 55, 1-7.

Cohen, E.A., Terwilliger, E.F., Sodroski, J.G., and Haseltine, W.A. (1988). Identification of a protein encoded by the Vpu gene of HIV-1. Nature 334, 532-534.

Cook, G.A., Zhang, H., Park, S.H., Wang, Y., and Opella, S.J. (2011). Comparative NMR studies demonstrate profound differences between two viroporins: $\mathrm{p} 7$ of HCV and Vpu of HIV-1. Biochim. Biophys. Acta Biomembr. 1808, 554-560.

Cordes, F.S., Tustian, A.D., Sansom, M.S.P., Watts, A., and Fischer, W.B. (2002). Bundles consisting of extended transmembrane segments of Vpu from HIV-1: computer simulations and conductance measurements. Biochemistry 41, 7359-7365.

De Angelis, A.A., Nevzorov, A.A., Park, S.H., Howell, S.C., Mrse, A.A., and Opella, S.J. (2004). High-resolution NMR spectroscopy of membrane proteins in aligned bicelles. J. Am. Chem. Soc. 126, 15340-15341.

Delaglio, F., Grzesiek, S., Vuister, G.W., Zhu, G., Pfeifer, J., and Bax, A. (1995). NMRPipe: a multidimensional spectral processing system based on UNIX pipes. J. Biomol. NMR 6, 277-293.

Dube, M., Bego, M.G., Paquay, C., and Cohen, E.A. (2010). Modulation of HIV-1-host interaction: role of the Vpu accessory protein. Retrovirology 7, 114.

Fares, C., Qian, J., and Davis, J.H. (2005). Magic angle spinning and static oriented sample NMR studies of the relaxation in the rotating frame of membrane peptides. J. Chem. Phys. 122, 194908-194917.

Fischer, W.B., Wang, Y.-T., Schindler, C., and Chen, C.-P. (2012). Mechanism of function of viral channel proteins and implications for drug development. Int. Rev. Cell Mol. Biol. 294, 259-321.

Fung, B.M., Khitrin, A.K., and Ermolaev, K. (2000). An improved broadband decoupling sequence for liquid crystals and solids. J. Magn. Reson. 142, 97-101.

Goddard, T.D. and Kneller, D.G. SPARKY 3. University of California, San Francisco, CA.

Hartmann, S.R. and Hahn, E.L. (1962). Nuclear double resonance in rotating frame. Phys. Rev. 128, 2042-2053.

Heise, H., Köhler, F.H., and Xie, X.L. (2001). Solid-state NMR spectroscopy of paramagnetic metallocenes. J. Magn. Reson. 150, 198-206.

Hohwy, M., Rienstra, C.M., Jaroniec, C.P., and Griffin, R.G. (1999). Fivefold symmetric homonuclear dipolar recoupling in rotating solids: application to double quantum spectroscopy. J. Chem. Phys. 110, 7983-7992.

Hong, M., Zhang, Y., and Hu, F.H. (2012). Membrane protein structure and dynamics from NMR spectroscopy. Annu. Rev. Phys. Chem. 63, 1-24.

Huster, D., Yao, X.L., and Hong, M. (2002). Membrane protein topology probed by $\mathrm{H}-1$ spin diffusion from lipids using solid-state NMR spectroscopy. J. Am. Chem. Soc. 124, 874-883.

Kijac, A., Shih, A.Y., Nieuwkoop, A.J., Schulten, K., Sligar, S.G., and Rienstra, C.M. (2010). Lipid-protein correlations in nanoscale phospholipid bilayers determined by solid-state nuclear magnetic resonance. Biochemistry 49, 9190-9198.

Klimkait, T., Strebel, K., Hoggan, M.D., Martin, M.A., and Orenstein, J.M. (1990). The human immunodeficiency virus type 1-specific protein vpu is required for efficient virus maturation and release. J. Virol. 64, 621-629.

Kochendoerfer, G.G., Jones, D.H., Lee, S., Oblatt-Montal, M., Opella, S.J., and Montal, M. (2004). Functional character- ization and NMR Spectroscopy on full-length Vpu from HIV-1 prepared by total chemical synthesis. J. Am. Chem. Soc. 126, 2439-2446.

Kumashiro, K.K., Schmidt-Rohr, K., Murphy, O.J., Ouellette, K.L., Cramer, W.A., and Thompson, L.K. (1998). A novel tool for probing membrane protein structure: solid-state NMR with proton spin diffusion and X-nucleus detection. J. Am. Chem. Soc. 120, 5043-5051.

Lenburg, M.E. and Landau, N.R. (1993). Vpu-induced degradation of $C D 4$ - requirement for specific amino-acid-residues in the cytoplasmic domain of CD4. J. Virol. 67, 7238-7245.

Lewis, B.A., Harbison, G.S., Herzfeld, J., and Griffin, R.G. (1985). NMR structural-analysis of a membrane-protein-bacteriorhodopsin peptide backbone orientation and motion. Biochemistry 24, 4671-4679.

Litman, B.J., Lewis, E.N., and Levin, I.W. (1991). Packing characteristics of highly unsaturated bilayer lipids: Raman spectroscopic studies of multilamellar phosphatidylcholine dispersions. Biochemistry 30, 313-319.

Lowe, I.J. (1959). Free induction decays of rotating solids. Phys. Rev. Lett. 2, 285-287.

Lu, J.X., Sharpe, S., Ghirlando, R., Yau, W.M., and Tycko, R. (2010). Oligomerization state and supramolecular structure of the HIV-1 Vpu protein transmembrane segment in phospholipid bilayers. Protein Sci. 19, 1877-1896.

Luca, S., Filippov, D.V., van Boom, J.H., Oschkinat, H., de Groot, H.J.M., and Baldus, M. (2001). Secondary chemical shifts in immobilized peptides and proteins: A qualitative basis for structure refinement under Magic Angle Spinning. J. Biomol. NMR 20, 325-331.

Luis Nieva, J., Madan, V., and Carrasco, L. (2012). Viroporins: structure and biological functions. Nat Rev Microbiol 10 , 563-574.

Ma, C., Marassi, F.M., Jones, D.H., Straus, S.K., Bour, S., Strebel, K., Schubert, U., Oblatt-Montal, M., Montal, M., and Opella, S.J. (2002). Expression, purification, and activities of full-length and truncated versions of the integral membrane protein Vpu from HIV-1. Protein Sci. 11, 546-557.

Maddon, P.J., Littman, D.R., Godfrey, M., Maddon, D.E., Chess, L., and Axel, R. (1985). The isolation and nucleotide-sequence of a cDNA encoding the T-cell surface protein T4 - a newer member of the immunoglobulin gene family. Cell 42, 93-104.

Maddon, P.J., Molineaux, S.M., Maddon, D.E., Zimmerman, K.A., Godfrey, M., Alt, F.W., Chess, L., and Axel, R. (1987). Structure and expression of the human and mouse T4 genes. Proc. Natl. Acad. Sci. USA 84, 9155-9159.

Magadan, J.G. and Bonifacino, J.S. (2012). Transmembrane domain determinants of CD4 downregulation by HIV-1 Vpu. J. Virol. 86, 757-772.

Magadan, J.G., Perez-Victoria, F.J., Sougrat, R., Ye, Y., Strebel, K., and Bonifacino, J.S. (2010). Multilayered mechanism of CD4 downregulation by HIV-1 Vpu involving distinct ER retention and ERAD targeting steps. PLoS Pathog. 6, e1000869.

Maldarelli, F., Chen, M.Y., Willey, R.L., and Strebel, K. (1993). Human-immunodeficiency-virus type- 1 Vpu protein is an oligomeric type-l integral membrane-protein. J. Virol. 67, 5056-5061.

Marassi, F.M., Das, B.B., Lu, G.J., Nothnagel, H.J., Park, S.H., Son, W.S., Tian, Y., and Opella, S.J. (2011). Structure determination of membrane proteins in five easy pieces. Methods 55, 363-369. 
Margottin, F., Benichou, S., Durand, H., Richard, V., Liu, L.X., Gomas, E., and Benarous, R. (1996). Interaction between the cytoplasmic domains of HIV-1 Vpu and CD4: role of Vpu residues involved in CD4 interaction and in vitro CD4 degradation. Virology 223, 381-386.

McDermott, A. (2009). Structure and dynamics of membrane proteins by magic angle spinning solid-state NMR. Annu. Rev. Biophys. 38, 385-403.

Miao, Y., Qin, H., Fu, R., Sharma, M., Can, T.V., Hung, I., Luca, S., Gor'kov, P.L., Brey, W.W., and Cross, T.A. (2012). M2 proton channel structural validation from full-length protein samples in synthetic bilayers and E. coli membranes. Angew. Chem. Int. Ed. 51, 8383-8386.

Morris, G.A. and Freeman, R. (1979). Enhancement of nuclear magnetic-resonance signals by polarization transfer. J. Am. Chem. Soc. 101, 760-762.

Neil, S.J.D., Zang, T., and Bieniasz, P.D. (2008). Tetherin inhibits retrovirus release and is antagonized by HIV-1 Vpu. Nature 451, 425-U421.

Page, R.C., Li, C., Hu, J., Gao, F.P., and Cross, T.A. (2007). Lipid bilayers: an essential environment for the understanding of membrane proteins. Magn. Reson. Chem. 45, S2-S11.

Park, S.H. and Opella, S.J. (2005). Tilt angle of a trans-membrane helix is determined by hydrophobic mismatch. J. Mol. Biol. 350, 310-318.

Park, S.H. and Opella, S.J. (2007). Conformational changes induced by a single amino acid substitution in the trans-membrane domain of Vpu: Implications for HIV-1 susceptibility to channel blocking drugs. Protein Sci. 16, 2205-2215.

Park, S.H., Mrse, A.A., Nevzorov, A.A., Mesleh, M.F., Oblatt-Montal, M., Montal, M., and Opella, S.J. (2003). Three-dimensional structure of the channel-forming trans-membrane domain of virus protein "u" (Vpu) from HIV-1. J. Mol. Biol. 333, 409-424.

Park, S.H., De Angelis, A.A., Nevzorov, A.A., Wu, C.H., and Opella, S.J. (2006a). Three-dimensional structure of the transmembrane domain of Vpu from HIV-1 in aligned phospholipid bicelles. Biophys J. 91, 3032-3042.

Park, S.H., Mrse, A.A., Nevzorov, A.A., De Angelis, A.A., and Opella, S.J. (2006b). Rotational diffusion of membrane proteins in aligned phospholipid bilayers by solid-state NMR spectroscopy. J. Magn. Reson. 178, 162-165.

Pines, A., Gibby, M.G., and Waugh, J.S. (1973). Proton-enhanced NMR of dilute spins in solids. J. Chem. Phys. 59, 569-590.

Schubert, U., Bour, S., FerrerMontiel, A.V., Montal, M., Maldarelli, F., and Strebel, K. (1996a). The two biological activities of human immunodeficiency virus type $1 \mathrm{Vpu}$ protein involve two separable structural domains. J. Virol. 70, 809-819.

Schubert, U., FerrerMontiel, A.V., OblattMontal, M., Henklein, P., Strebel, K., and Montal, M. (1996b). Identification of an ion channel activity of the Vpu transmembrane domain and its involvement in the regulation of virus release from HIV-1-infected cells. FEBS Lett. 398, 12-18.

Seidel, K., Lange, A., Becker, S., Hughes, C.E., Heise, H., and Baldus, M. (2004). Protein solid-state NMR resonance assignments from (C-13, C-13) correlation spectroscopy. Phys. Chem. Chem. Phys. 6, 5090-5093.

Sharpe, S., Yau, W.M., and Tycko, R. (2006). Structure and dynamics of the HIV-1 Vpu transmembrane domain revealed by solid-state NMR with magic-angle spinning. Biochemistry 45 , 918-933.
Singh, S.K., Mockel, L., Thiagarajan-Rosenkranz, P., Wittlich, M., Willbold, D., and Koenig, B.W. (2012). Mapping the interaction between the cytoplasmic domains of HIV-1 viral protein $\mathrm{U}$ and human CD4 with NMR spectroscopy. FEBS J. 279, 3705-3714.

Strebel, K., Klimkait, T., and Martin, M.A. (1988). A novel gene of HIV-1, Vpu, and its 16-kilodalton product. Science 241, 1221-1223.

Strebel, K., Klimkait, T., Maldarelli, F., and Martin, M.A. (1989). Molecular and biochemical analyses of human immunodeficiency virus type 1 vpu protein. J. Virol. 63, 3784-3791.

Szeverenyi, N.M., Sullivan, M.J., and Maciel, G.E. (1982). Observation of spin exchange by two-dimensional Fouriertransform C-13 cross polarization magic-angle spinning. J. Magn. Reson. 47, 462-475.

Tiganos, E., Yao, X.J., Friborg, J., Daniel, N., and Cohen, E.A. (1997). Putative $\alpha$-helical structures in the human immunodeficiency virus type $1 \mathrm{Vpu}$ protein and CD4 are involved in binding and degradation of the CD4 molecule. J. Virol. 71, 4452-4460.

Van Damme, N., Goff, D., Katsura, C., Jorgenson, R.L., Mitchell, R., Johnson, M.C., Stephens, E.B., and Guatelli, J. (2008). The interferon-induced protein BST-2 restricts HIV-1 release and is downregulated from the cell surface by the viral Vpu protein. Cell Host Microbe 3, 245-252.

Vincent, M.J., Raja, N.U., and Jabbar, M.A. (1993). Human-immunodeficiency-virus type-1 Vpu protein induces degradation of chimeric envelope glycoproteins bearing the cytoplasmic and anchor domains of CD4 - role of the cytoplasmic domain in $\mathrm{Vpu}$-induced degradation in the endoplasmic-reticulum. J. Virol. 67, 5538-5549.

Wei, Y., Lee, D.-K., and Ramamoorthy, A. (2001). Solid-state $13 \mathrm{C}$ NMR chemical shift anisotropy tensors of polypeptides. J. Am. Chem. Soc. 123, 6118-6126.

Willey, R.L., Bucklerwhite, A., and Strebel, K. (1994). Sequences present in the cytoplasmic domain of CD4 are necessary and sufficient to confer sensitivity to the human-immunodeficiencyvirus type-1 Vpu protein. J. Virol. 68, 1207-1212.

Wittlich, M., Koenig, B.W., Hoffmann, S., and Willbold, D. (2007a). Structural characterization of the transmembrane and cytoplasmic domains of human CD4. Biochim. Biophys. Acta Biomembr. 1768, 2949-2960.

Wittlich, M., Wiesehan, K., Koenig, B.W., and Willbold, D. (2007b). Expression, purification, and membrane reconstitution of a CD4 fragment comprising the transmembrane and cytoplasmic domains of the receptor. Protein Expr. Purif. 55, 198-207.

Wittlich, M., Koenig, B.W., Stoldt, M., Schmidt, H., and Willbold, D. (2009). NMR structural characterization of HIV-1 virus protein U cytoplasmic domain in the presence of dodecylphosphatidylcholine micelles. FEBS J. 276, 6560-6575.

Wittlich, M., Thiagarajan, P., Koenig, B.W., Hartmann, R., and Willbold, D. (2010). NMR structure of the transmembrane and cytoplasmic domains of human CD4 in micelles. Biochim. Biophys. Acta Biomembr. 1798, 122-127.

Yang, J., Aslimovska, L., and Glaubitz, C. (2011). Molecular dynamics of proteorhodopsin in lipid bilayers by solid-state NMR. J. Am. Chem. Soc. 133, 4874-4881.

Yao, X.J., Friborg, J., Checroune, F., Gratton, S., Boisvert, F., Sekaly, R.P., and Cohen, E.A. (1995). Degradation of CD4 induced by human-immunodeficiency-virus type-1 Vpu protein - a predicted alpha-helix structure in the proximal cytoplasmic region of CD4 contributes to Vpu sensitivity. Virology 209, 615-623. 